UDC 616.33

\title{
ROLE OF HELICOBACTER PYLORI AND HOST GENE POLYMORPHISMS IN GASTRIC CARCINOGENESIS IN A KAZAKHS
}

\author{
Kulmambetova G.N., ${ }^{1}$ Imanbekova M.K., ${ }^{1}$ Aitkulova A.M., ${ }^{1}$ Shtefanov I.I., ${ }^{2}$ \\ Makishev A.K., ${ }^{2}$ Ramankulov Ye.M. ${ }^{1}$ \\ ${ }^{1}$ National Center for Biotechnology \\ 13/5, Korgalzhyn highway, Astana, 010000, Kazakhstan \\ ${ }^{2}$ Oncology Center of Astana \\ 17, Manas str., Astana, 010000, Kazakhstan \\ gulmirakn@gmail.com
}

\begin{abstract}
Emerging evidence indicated that common polymorphisms of TNF- $\alpha$, IL1B, IL10, TP53, CD14, TLR4 genes might impact individual susceptibility to gastric cancer. Moreover, Helicobacter pylori plays an important role, provoking an increased susceptibility to the development of gastric pathologies. However, the results are still inconclusive, and specific alleles vary in different populations. We conducted a study in the Kazakh population of the Northern region of Kazakhstan to identify potential risks or protective associations of SNP with gastric cancer, or $\mathrm{H}$. pylori infection. We analyzed the association of SNPs in TNF-a (rs1800629 G/A), IL1B (rs16944 A/G), IL10 (rs1800872 G/T, rs1800871 G/A, rs1800896 A/C), TP53 (rs1042522 A/G), CD14 (rs2569190 G/A), and TLR4 (rs4986790 A/G, rs4986791 C/T) genes. We included cases with gastric cancer in a hospital-based Kazakh population (158 cases and 181 cancer-free controls). Identification of Helicobacter pylori was carried out by microbiological methods from biopsy specimens. Genotyping of the extended panel of polymorphisms of candidate genes was performed on the Quant Studio 12K Flex (Life Technologies). To estimate relative risk, odds ratio (OR) and $95 \%$ confidence interval (CI) were calculated, and $P$ value 0.05 was considered statistically significant. We found that the polymorphisms of TP53 (rs1042522 A/G, OR: 2.32, 95\% CI: 1.41-3.82, P-value=0.0009), CD14 (rs2569190 G/A, OR: 0.59, 95\% CI: 0.36 - 0.96, P-value=0.035), and TLR4 (rs4986790 A/G, OR: 0.43, 95\% CI: $0.19-\mathbf{0 . 9 5}$, P-value=0.038) are associated with gastric cancer risk. No significant association was observed between variant genotype polymorphisms of TNF- $\alpha$, IL1B, IL10, TLR4 (ID: rs4986791) genes and gastric cancer risk. Further analyzes to assess the combined effect of TP53 rs1042522, CD14 rs2569190 and TLR4 rs4986791 and $H$. pylori infection on GC risk did not reveal significant associations. Our results testify to markers of GC risk among Kazakhs and may be the first step towards new approaches to diagnosis and prognosis in Kazakhstan.
\end{abstract}

Keywords: gastric cancer, Helicobacter pylori, polymorphism, cytokine, Kazakh

Gastric cancer (GC) is the third most common cause of mortality in the world. According to the annual statistical data "Indicators of the cancer service of the Republic of Kazakhstan", the mortality rate from gastric cancer in the Republic of Kazakhstan for 2016 is 9.8 cases per 100 thousand of population, the incidence is 15.4 cases per 100 thousand of population. The problem of gastric cancer for our country is very relevant. The International Agency for Research on Cancer, after numerous studies, officially included as the etiological factor of the bacterium Helicobacter pylori. The bacterium initiates chronic inflammation in the gastric mucosa, mediating the production of pro- and anti-inflammatory cytokines in the host organism [1]. The intensity of the cytokine response depends on the genetic polymorphism of the genes encoding them, which determines the clinical picture of the development of the disease. Therefore, as an important factor in the development of the disease is the genetics of the individual [2].

Growing studies explored the association of polymorphisms in TNF- $\alpha$, IL1B, IL10, TP53, CD14, TLR4 genes with susceptibility to gastric cancer, but the results have remained controversial and conflicting. However, all these studies are conducted in Caucasian and Asian population, especially Chinese and Japanese. In Kazakhstan no particular study was interested in the association between polymorphisms of the genes and high risk of cancers. In the present study, to explore whether these polymorphisms were associated with risks of gastrointestinal cancers, we performed a case-control study on the association between the nine most frequently 
studied polymorphisms (rs1800629 G/A, rs16944 A/G, rs1800872 G/T, rs1800871 G/A, rs1800896 A/C, rs2569190 G/A, rs1042522 A/G, rs4986790 A/G, rs4986791 C/T) and cancer risk in a Kazakhs.

\section{MATERIALS AND METHODS}

\section{Study subjects}

A clinical-based case-control study was conducted in 158 cases (patients suffering from GC) and 181 controls (healthy volunteers, without any gastric disorders). Patients with gastric disorders were identified based on a pathologic diagnosis at the Oncology center of Astana. The control group and the study group were recruited from the same geographical area (North Kazakhstan region). Controls were frequency matched to cases by age at enrollment ( \pm 6 years).

\section{Ethical statement}

A comprehensive set of clinical, laboratory and sociodemographic (age, gender, and occupation), history of smoking and alcohol drinking, family history of cancer and frequency of food consumption data have been collected. Prior to the study, ethical approval was received from the Ethics Committee of the National Center for Biotechnology (№2 12.03.2012). The Ethics Committee approved the informed consent form and questionnaire form designed specifically for the study. The investigation was conducted according to all current ethical guidelines. Written informed consent was obtained for all subjects and blood sample donation. $9 \mathrm{ml}$ venous blood sample was collected from each subject after the interview.

\section{Identification of Helicobacter pylori} specimens.

Detection of bacterium Helicobacter pylori was performed by microbiological methods from biopsy

\section{DNA extraction} $70{ }^{\circ} \mathrm{C}$ until use [3].

Genomic DNA was extracted from venous blood samples using salting-out method, and then stored at -

\section{SNP genotyping}

Genotyping of the extended panel of polymorphisms of candidate genes was performed on the Quant Studio 12K Flex (Life Technologies). The total reaction volume was $5 \mu$, containing $2.5 \mu \mathrm{l}$ of $2 \mathrm{x}$ Oppen Array Real-time master mix and $2.5 \mu \mathrm{l}$ of DNA with concentration $50 \mathrm{ng} / \mu \mathrm{l}$. Temperature conditions: $93{ }^{\circ} \mathrm{C}$ for 10 min; amplification was followed by 50 cycles at $93{ }^{\circ} \mathrm{C}$ for $45 \mathrm{~s}, 94^{\circ} \mathrm{C}$ for $13 \mathrm{~s}, 53.5^{\circ} \mathrm{C}$ for $2.14 \mathrm{~min}$, and a final incubation at $25{ }^{\circ} \mathrm{C}$ for $2 \mathrm{~min}$. Data analysis was performed using the software package TaqMan Genotyper Software v.1.3.

\section{Statistical analyses}

Category variables and Hardy-Weinberg equilibrium (HWE) in genotypic distributions were assessed by chi-square $(\chi 2)$ tests. Association between genetic polymorphism and the risk of cancers was estimated by unconditional logistic regression analysis. To estimate relative risk, odds ratio (OR) and 95\% confidence interval (CI) were calculated, and P value 0.05 was considered statistically significant. Statistical analysis was performed using R (URL http://www.R-project.org/).

\section{RESULTS}

The distribution of each of the nine SNPs genotyped in the gastric cancer group fitted the HardyWeinberg equilibrium law except for TP53 rs1042522. The major allele homozygotes in all the SNPs were used as the reference genotypes.

The TP53 rs 1042522 Arg72Pro G allele markedly increased the risk of gastric disorders in these ethnic Kazakh individuals showing ORs of 2.32 (95\% CI: 1.41-3.82, P-value=0.0009). Individuals harbouring the CD14 rs2569190 -260 A allele (OR: 0.59, 95\% CI: $0.36-0.96$, P-value $=0.035$ ) was found to be significantly protected against the development of gastric disorders in Kazakhs. Although the CD14 -260 A allele showed a tendency towards protection against GC, this was not statistically significant (OR: 0.87, 95\% CI: 0.63-1.21, Pvalue $=0.41)$. The TLR4 rs4986790 $-896 \mathrm{G}$ allele was found to be also significantly protected against the development of GC (OR: 0.43, 95\% CI: 0.19 - 0.95, P-value $=0.038$ ) in a Kazakhs. No significant associations were found between TNF- $\alpha$, IL1B, IL10, TLR4 (ID: rs4986791) in our case-control study.

Given that $H$. pylori is known to be major risk factor associated with GC, further analyses were performed to assess the joint effect of TP53 rs1042522, CD14 -260 G/A and TLR4 rs4986790 and H. pylori infection on risk of GC. To confirm that no association existed between TP53 rs1042522, CD14 -260 G/A and TLR4 rs4986790 and risk of $H$. pylori infection, univariate statistical analyses were conducted, showing non significant results (Table 1). 
Table 1. Association between TP53, CD14 and TLR4 polymorphisms and risk of Helicobacter pylori in ethnic Kazakh individuals

\begin{tabular}{|llllll|}
\hline & \multicolumn{5}{c|}{ H. pylori } \\
Polymorphism & Genotype/Allele & $\begin{array}{l}\text { H. pylori } \\
\text { positive (N) } \\
\text { negative (N) }\end{array}$ & OR (95\%CI) & P value \\
\hline rs1042522 Arg72Pro & CC & 29 & 29 & & \\
& GC & 30 & 46 & & \\
& GG & 7 & 10 & & \\
& C & 88 & 104 & 1 & 0.3258 \\
& G & 44 & 66 & $0.78(0.4897-1.2676)$ & \\
rs2569190 -260C/T & GG & 20 & 19 & & \\
& AG & 29 & 42 & & \\
& AA & 17 & 24 & & \\
& G & 69 & 80 & 1 & \\
& A & 63 & 90 & $0.81(0.5147-1.2797)$ & \\
rs4986790 -896A/G & AA & 42 & 49 & & \\
& AG & 3 & 4 & & \\
& GG & 0 & 0 & & \\
& A & 87 & 102 & 1 & \\
& G & 3 & 4 & $0.88(0.1915-4.0368)$ & \\
& & & & & \\
\end{tabular}

Genetic susceptibility genes for gastric cancer play very important roles in the development and progression of gastric cancer [4]. Although the pathogenesis and genetic mechanisms of several genes have been elucidated recently, further large-sample studies are required after the meta-analysis of many genes. In addition, different populations in different regions with different races will have certain differences in genetic susceptibility [5]. Therefore, further in-depth studies are needed to search for gene therapy methods to eventually provide a scientific basis for the prevention and control of gastric cancer. We found that the polymorphisms of TP53 (rs1042522) is associated with gastric cancer risk and CD14 (rs2569190), TLR4 (rs4986790) were found as protection against developing GC, whereas no significant association was observed between variant genotype of other six polymorphisms (TNF- $\alpha$ rs1800629 G/A, IL-1B rs16944 A/G, IL-10 rs1800872 G/T, IL-10 rs1800871 G/A, IL-10 rs1800896 A/C, TLR4 rs4986791 C/T) and gastric cancer risk.

In summary, our case-control study observed the significant association of TP53 (rs1042522) with gastric cancer risk. Furthermore, we found the association between CD14 (rs2569190) and TLR4 (rs4986790) and gastric cancer was also significant in Kazakhs. Our results indicated to markers of GC risk among Kazakhs and may be the first step towards new approaches to diagnosis and prognosis in Kazakhstan.

\section{Funding}

This study was funded by the Ministry of Education and Science of the Republic of Kazakhstan by O.0584 STP "Creation of genetic passports for certain groups of the population of Kazakhstan based on the using DNA technology" for 2012-2014 years.

\section{REFERENCES}

1. Uemura N, Okamoto S, Yamamoto S, Matsumura N, Yamaguchi S, Yamakido M, et al. Helicobacter pylori infection and the development of gastric cancer. N Engl J Med. 2001;345(11):784-9.

2. Kamangar F, Cheng C, Abnet CC, Rabkin CS. Interleukin-1B polymorphisms and gastric cancer risk--a metaanalysis. Cancer Epidemiol Biomarkers Prev. 2006;15(10):1920-8.

3. Miller SA, Dykes DD, Polesky HF. A simple salting out procedure for extracting DNA from human nucleated cells. Nucleic Acids Res. 1988;16(3).

4. Kulmambetova GN, Imanbekova MK, Logvinenko AA, Sukashev AT, Filipenko ML, Ramanculov EM. Association of cytokine gene polymorphisms with gastritis in a Kazakh population. Asian Pac J Cancer Prev. 2014;15(18):7763-8. 
5. Ma J, Wu D, Hu X, Li J, Cao M, Dong W. Associations between cytokine gene polymorphisms and susceptibility to Helicobacter pylori infection and Helicobacter pylori related gastric cancer, peptic ulcer disease: A meta-analysis. PLoS One. 2017;12(4). 